Exp. Anim. 56(1), 11-20, 2007

\title{
Severe Insulin Resistance and Moderate Glomerulosclerosis in a Minipig Model Induced by High-Fat/ High-Sucrose/ High-Cholesterol Diet
}

\author{
Yi LIU' ${ }^{1,3)}$, Zongbao WANG'), Weidong YIN'1), Qinkai ${ }^{11}{ }^{1)}$, Manbo CAI ${ }^{1)}$, \\ Chi ZHANG ${ }^{1)}$, Junxia XIAO'), Hongjie HOU'1), Hongguang ${ }^{1}{ }^{1)}$, and Xiuhong ZU' ${ }^{1)}$

\begin{abstract}
1)Institute of Cardiovascular Research, School of Life Sciences and Technology, ${ }^{2) D e p a r t m e n t ~ o f ~}$ of Shaoguan University, Shaoguan, Guangdong 512026, P. R. China
\end{abstract} \\ Laboratory Animal Science, Nanhua University, Hengyang, Hunan 421001, and ${ }^{3)}$ Medical College
}

\begin{abstract}
To develop a minipig model of type 2 diabetes that simulates the common manifestations of the metabolic abnormalities and resembles the kidney pathology of type 2 diabetes in the human population, male Chinese Bama minipigs were divided into 2 groups (5 in each) and fed with a control diet (CD) or high-fat/ high-sucrose/ high-cholesterol diet (HFSCD) for 5 months. The biochemical parameters of blood and urine, and the oral glucose tolerance test were monitored after the feeding program. The insulin resistance was estimated by the HOMA-IR index and the glucose elimination constant $\left(K_{G}\right)$, and betacell function by the HOMA-beta index and the acute insulin response (AIR). Glomerulosclerosis index (GSI) was semi-quantitated by the degree of glomerular lesions in kidney sections stained with Masson trichrome. Extracellular matrix deposition in the kidney was examined by the protein expression of type IV collagen, connective tissue growth factor (CTGF) and matrix metalloproteinases 2 (MMP-2) using immunohistochemistry. Feeding HFSCD to minipigs markedly caused hyperglycaemia, hyperinsulinaemia and dyslipidaemia. HOMA-IR was significantly increased while HOMAbeta, AIR and $K_{G}$ were obviously decreased in the HFSCD group compared with control group. Microalbuminuria, glucosuria and moderate glomerulosclerosis were exhibited in HFSCD-fed minipigs. The expression of type IV collagen and CTGF was elevated whereas that of MMP-2 was reduced in the kidneys of HFSCD group compared with the CD group. We concluded that feeding HFSCD to Chinese Bama minipigs for 5 months can induce humanoid type 2 diabetes and early-stage diabetic nephropathy, and accelerate extracellular matrix deposition and glomerulosclerosis.
\end{abstract}

Key words: diabetic nephropathy, diabetogenic diet, extracellular matrix, minipig model, type 2 diabetes 


\section{Introduction}

Although type 2 diabetes is ten times more prevalent than type 1 diabetes, the progression of renal disease in type 2 diabetes has been studied less intensively and is not as well understood. Type 2 diabetes, unlike type 1 diabetes, is a more insidious disorder. Peripheral insulin resistance exacerbates and blood insulin increases to compensate for the insulin resistance. Hyperglycaemia and islet beta-cell degeneration take place progressively. Microalbuminuria may occur during the phase of insulin resistance. However, for ethical reasons, the pathogenesis of diabetic nephropathy during this phase cannot be studied with biopsies. In view of the incompletely understood pathogenesis, additional studies on nephropathy related to type 2 diabetes are required. Most animal models do not fulfill study requirements, since they are based on monogenic disorders of little relevance to human diabetes or on chemical destruction of beta-cells, which is also less clinically relevant [19]. It has been shown by several groups that type 2 diabetes can be induced in the mouse [19], rat [5], hamster [16] and minipigs $[8,20]$ by diabetogenic diet enriched in high-fat and high-energy. High saturated fat also induced type 2 diabetes and diabetic nephropathy in C57BL/6J mice [17] and Golden Syrian hamsters [12]. Diet-induced hypercholesterolaemia developed renal injury in rats and domestic crossbred pigs $[6,18]$. These models are more similar to the human condition and can be used in studies on the pathophysiology of type 2 diabetes and renal complications.

To our knowledge, the current study is the first to demonstrate that a high-fat/ high-sucrose/ high-cholesterol diet induces renal structural and functional changes similar to human type 2 diabetes in Chinese Bama minipigs. Chinese Bama minipigs are a characteristic strain of miniature pigs in China which have been produced by inbreeding of half-siblings, resulting in the animal's characteristic small size, stable genetics and uniform phenotype of "two black ends" in which the fur of the head and rump is black and that of the rest of the body is white.

\section{Materials and Methods}

\section{Animal and diets}

Ten male Chinese Bama miniature pigs, 3 months of age, were obtained from the Laboratory Animal Center of the Third Military Medical University (Chongqing, China). A cohort of animals was randomized into 2 groups of similar body weight: $n=5$ in the normal control diet (CD) group; $n=5$ in the high-fat/ high-sucrose/ high-cholesterol diet (HFSCD) group. The HFSCD used in this study was $51 \%$ normal miniature pig's diet supplemented with $10 \%$ lard, $37 \%$ sucrose and $2 \%$ cholesterol [20]. On a caloric basis, the HFSCD consisted of $38.26 \%$ fat from lard, $53.52 \%$ carbohydrate, and $8.22 \%$ protein (total $21.55 \mathrm{MJ} / \mathrm{kg}$ ), whereas the normal diet contained $10.35 \%$ fat, $68.99 \%$ carbohydrate, and $20.66 \%$ protein (total $13.13 \mathrm{MJ} / \mathrm{kg}$ ). The amount of daily fodder was $4 \%$ weight of minipigs which were fed three times daily with either CD or HFSCD. Water was available ad libitum. Body weights were recorded every two weeks. The total study period was 5 months. All experiments were performed according to the "Principles of Laboratory Animal Care" (NIH publication no. 85-23, revised 1985).

\section{Biochemical assays}

Blood samples were obtained from the orbital sinus of the animals at the end of each month after an overnight fast, and the plasma specimens were used for measuring glucose, insulin, triglycerides, total cholesterol, creatinine, blood urea nitrogen (BUN) and $\mathrm{N}$-acetyl-beta-glucosaminidase (NAG). Glucose, triglycerides and total cholesterol were determined by enzymatic kits (Rongsheng Biotech., Shanghai, China). Insulin was assayed by a radioimmunoassay kit (China Institute of Atomic Research, Beijing, China).

The homeostatic model assessment (HOMA) is widely used to assess longitudinal changes in beta cell function and insulin resistance (IR) in order to examine the natural history of diabetes. The following equations are used in HOMA: Homa-beta $=(20 \times$ FINS $) /$ $($ FPG-3.5) [15], Homa-IR $=($ FPG $\times$ FINS $) / 22.5$ [2] for beta cell function and IR, respectively, where FINS is the fasting plasma insulin concentration (U/l) and FPG is fasting plasma glucose ( $\mathrm{mmol} / \mathrm{l})$.

Randomly obtained urine samples in the morning were used for determination of glucose, microalbumin, creatinine, BUN and NAG at months 0, 2, 4 and 5 . Urinary microalbumin was determined using a turbilatex-based immunoassay. Glucose in the urine was assayed by the hexokinase method [7] (albumin 
and glucose kits from Mingdian Bio., Shanghai, China). Plasma and urinary creatinine, BUN and NAG were measured using the Jaffe, Fearon and PNP methods, respectively (kits from Jianchen Biotech., Nanjing, China). The urinary glucose/creatinine ratio, albumin/ creatinine ratio [11] and NAG/creatinine ratio [1] were calculated. The NAG clearance, expressed as per cent of creatinine clearance, was calculated according to the formula: (urinary NAG/plasma NAG) $\times$ (plasma creatinine/urinary creatinine) $\times 100$.

\section{Oral glucose tolerance test (OGTT)}

For assessment of glucose tolerance, an OGTT was performed on the minipigs at monthly intervals. After an 18-h overnight fast, animals were given $2 \mathrm{~g} / \mathrm{kg}$ Dglucose by gavage through a gastric tube which was inserted into the stomach. Blood samples were obtained to determine plasma glucose and insulin via the auricular veins at $0,15,30,60,90$, and $120 \mathrm{~min}$ after glucose administration.

In the OGTT, the acute insulin response (AIR) was estimated as the mean of the increase in plasma insulin at $15 \mathrm{~min}$ above the baseline, and the glucose elimination was quantified as $K_{G}$ (i.e., the glucose elimination constant), the slope of the natural logarithm of the glucose values at 15, 30 and 60 min after the glucose load, and expressed as percent elimination of glucose per minute [1].

\section{Pancreatic islet histopathology}

To examine the effect of the diet on pancreatic islets, samples of pancreas were fixed in phosphate-buffered $10 \%$ formalin ( $\mathrm{pH} \mathrm{7.4)}$ for $24 \mathrm{~h}$, paraffin-embedded, sectioned $(4 \mu \mathrm{m})$ and stained with hemotoxylin and $\operatorname{eosin}(\mathrm{H} \& \mathrm{E})$.

\section{Kidney histopathology and semiquantitation}

Each left kidney removed from anesthetized minipigs was weighed and the kidney weight index (left kidney weight/body weight ratio, $\mathrm{g} / \mathrm{kg}$ ) was calculated at the end of month 5. Left kidneys were sliced transversely in the same place, fixed in $10 \%$ formaldehyde in 0.1 mol/l PBS (pH 7.4), embedded in paraffin, and sectioned at $4 \mu \mathrm{m}$. Sections stained with H\&E and Masson trichrome were used to evaluate the general histological changes in glomerular structure.

In kidney sections stained with Masson trichrome, at least 50 glomeruli from each minipig were randomly examined. The degree of lesions in each glomerulus was graded on a scale of 0 to $4+$ by the method of Saito et al [13]. Grade 0 represents a normal glomerulus; grade 1, 1-25\% loss of capillaries in the tuft (minimal sclerosis); grade 2, 26-50\% loss (moderate sclerosis); grade $3,51-75 \%$ loss (moderate-severe sclerosis) and grade $4,75-100 \%$ of the tuft sclerosed (severe sclerosis). An average score (glomerulosclerosis index, GSI) was calculated for each animal. Glomerulosclerosis was defined as GBM thickening, mesangial hypertrophy, hyalinosis, and capillary occlusion [4].

All sections were examined by technicians without prior knowledge of the experimental protocol.

\section{Immunohistochemistry}

Kidney sections were evaluated immunohistochemically using the EliVision ${ }^{\mathrm{TM}}$ plus horseradish peroxidase (HRP) System (Maixin Bio., Fuzhou, China). Briefly, deparaffinized and hydrated sections were pretreated with $0.1 \%$ trypsin for $30 \mathrm{~min}$ at room temperature. After quenching the endogenous peroxidase by immersion in $3 \%$ hydrogen peroxide for $10 \mathrm{~min}$, sections were incubated at $4{ }^{\circ} \mathrm{C}$ overnight with polyclonal antibodies against type IV collagen, connective tissue growth factor (CTGF) or matrix metalloproteinases 2 (MMP-2) (above primary antibodies from Boster Bio., Wuhan, China) diluted to 1:100 each. Then, the sections were incubated with polymer enhancer for $20 \mathrm{~min}$ and with polymerized HRP-labeled goat anti-rabbit IgG for 30 min at room temperature. The antigens were visualized using 3, 3'-diamino-benzidine chromogen solution. Finally, tissue sections were counterstained with hematoxylin, dehydrated and coverslipped for viewing. For all studies, negative controls were included with substitution of the primary antibodies with $10 \%$ non-immune goat serum.

\section{Statistical analysis}

All values are expressed as mean \pm SD. HOMA scores, urine glucose/creatinine ratio, albumin/creatinine ratio and NAG/creatinine ratio were log-transformed to approximate a normal distribution. Quantitative data were assessed by Student's $t$-test and semi-quantitative data were compared by Wilcoxon tests. Significance was accepted at the $P<0.05$ level. 


\section{Results}

\section{Effect of HFSCD on homeostasis}

Figure 1 shows that the body weights were similar in the two groups at the beginning of the experiment (CD $6.04 \pm 1.24 \mathrm{~kg} v s$. HFSCD $6.04 \pm 2.13 \mathrm{~kg}$ ), and linearly elevated with feeding duration. The weight gain was significantly higher in the HFSCD-fed minipigs than in CD-fed minipigs at week 18 and week $20(P<0.01)$.

As shown in Table 1, plasma glucose concentrations in the HFSCD group were linearly elevated with the time reaching $10.27 \pm 2.25 \mathrm{mmol} / \mathrm{l}$ at month 5 , which was 2.15 folds higher than that of the control group.

Insulin levels of HFSCD-fed minipigs rapidly elevated at month 2 and continued to increase to a peak of $24.43 \pm 3.55 \mathrm{U} / \mathrm{l}$ at month 4 in the HFSCD group, and dropped off thereafter.

Plasma triglyceride and total cholesterol concentrations were significantly increased from the first month of the experimentation, and the significant increase persisted for the study period in miniature pigs fed the HFSCD compared with those fed the control diet.

\section{Effect of HFSCD on glucose disposal and insulin se-} cretion

Table 2 shows the results of AIR and $K_{G}$ in the two groups of minipigs for OGTT performed every month. At 3 months after the start of HFSCD, miniature swine were already glucose intolerant and had impaired glucose-stimulated insulin secretion. Glucose elimination, as estimated by $K_{G}$, was $2.43 \pm 0.58 \% / \mathrm{min}$ in CD-fed pigs versus only $1.04 \pm 0.26 \% / \mathrm{min}$ in HFSCD-fed pigs $(P<0.05)$. Similarly, AIR was markedly lower in HFSCD-fed pigs $(24.39 \pm 6.17 \mathrm{U} / \mathrm{l})$ than in control diet-fed pigs $(43.73 \pm 8.40 \mathrm{U} / \mathrm{l} ; P<0.05)$. From the glucose clearance curve at the 5 th month of the experiment, the plasma glucose level was highest at $30 \mathrm{~min}$ and rapidly declined, approaching the starting values at 120 min for pigs of the CD group; hence, glucose removal for these pigs was efficient and rapid. In contrast, there was no glucose elimination but a gradual increase between 15 and $60 \mathrm{~min}$ in the glucose curve of the HFSCD group (HFSCD, $K_{G},-7.38 \pm 1.36 \% / \mathrm{min}$; CD, $1.95 \pm 0.21 \% / \mathrm{min})$, which suggests deficient glucose removal and severe glucose intolerance (Fig. 2a). The curve of plasma insulin levels exhibits that AIR to the

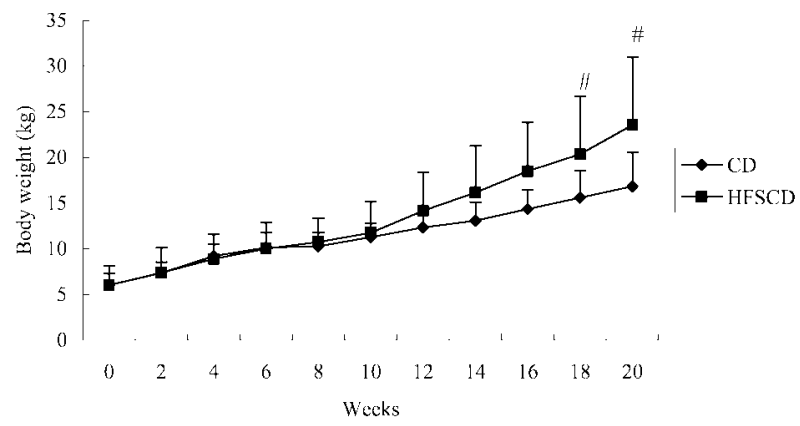

Fig. 1. Changes of the body weight in HFSCD- and CD-fed minipigs during the 5-month experimental period. Values are mean $\pm \mathrm{SD}$. ( $\# P<0.01$ vs. $\mathrm{CD}$ group)

Table 1. Effects of HFSCD on glucose and lipid metabolism parameters in Chinese Bama minipigs $(\mathrm{n}=5)$

\begin{tabular}{|c|c|c|c|c|c|c|}
\hline Parameters & 0 & 1 month & 2 months & 3 months & 4 months & 5 months \\
\hline \multicolumn{7}{|c|}{ Plasma glucose $(\mathrm{mmol} / \mathrm{l})$} \\
\hline $\mathrm{CD}$ & $4.71 \pm 0.39$ & $4.24 \pm 0.90$ & $4.83 \pm 0.87$ & $5.24 \pm 1.00$ & $4.65 \pm 0.71$ & $4.77 \pm 0.57$ \\
\hline HFSCD & $5.12 \pm 0.84$ & $5.97 \pm 0.73^{*}$ & $6.88 \pm 0.57 *$ & $8.89 \pm 2.05 \#$ & $9.54 \pm 2.12 \#$ & $10.27 \pm 2.25 \#$ \\
\hline \multicolumn{7}{|c|}{ Plasma insulin (U/l) } \\
\hline $\mathrm{CD}$ & $6.97 \pm 0.33$ & $7.31 \pm 0.94$ & $7.50 \pm 0.93$ & $8.68 \pm 0.82$ & $7.25 \pm 0.80$ & $7.65 \pm 1.33$ \\
\hline HFSCD & $7.47 \pm 1.16$ & $9.12 \pm 1.63$ & $15.12 \pm 3.22^{*}$ & $21.16 \pm 5.08 *$ & $24.43 \pm 3.55 \#$ & $17.43 \pm 3.80 \#$ \\
\hline \multicolumn{7}{|c|}{ Plasma triglycerides (mmol/l) } \\
\hline $\mathrm{CD}$ & $0.59 \pm 0.04$ & $0.59 \pm 0.06$ & $0.63 \pm 0.10$ & $0.69 \pm 0.16$ & $0.71 \pm 0.15$ & $0.73 \pm 0.13$ \\
\hline HFSCD & $0.59 \pm 0.05$ & $0.94 \pm 0.07 \#$ & $1.50 \pm 0.18 \#$ & $1.87 \pm 0.14 \#$ & $2.03 \pm 0.42 \#$ & $2.10 \pm 0.32 \#$ \\
\hline \multicolumn{7}{|c|}{ Plasma total cholesterol (mmol/l) } \\
\hline $\mathrm{CD}$ & $2.06 \pm 0.34$ & $1.99 \pm 0.33$ & $2.01 \pm 0.35$ & $1.68 \pm 0.21$ & $1.82 \pm 0.49$ & $2.07 \pm 0.46$ \\
\hline HFSCD & $2.31 \pm 0.44$ & $6.24 \pm 1.77 \#$ & $9.06 \pm 1.44 \#$ & $11.20 \pm 2.02 \#$ & $15.23 \pm 2.00 \#$ & $20.45 \pm 2.83 \#$ \\
\hline
\end{tabular}

Values are means $\pm \mathrm{SD} ; * P<0.05 \mathrm{HFSCD} v s . \mathrm{CD} ; \# P<0.01 \mathrm{HFSCD} v s . \mathrm{CD}$. 

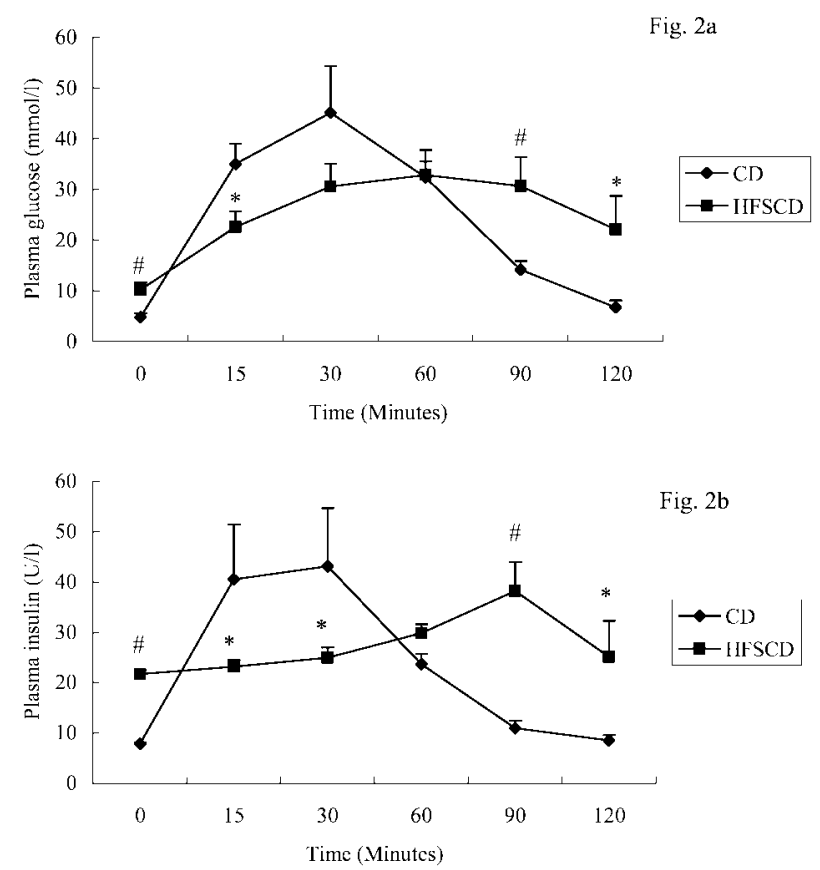

Fig. 2. Effects of the high-fat/high-sucrose/high-cholesterol diet fed to minipigs for 5 months on the levels of glucose (2a) and insulin (2b) in response to oral glucose loading examined by the oral glucose tolerance test. (2a) Pigs from the HFSCD group exhibited marked glucose intolerance compared with those from the CD group. (2b) The deficient glucose removal seen in the HFSCD group could be caused by impairment of acute insulin secretion in response to the glucose load. Values are mean \pm SD. $\left({ }^{*} P<0.05, \# P<0.01\right.$, HFSCD $\left.v s . \mathrm{CD}\right)$ oral glucose challenge was blunted after HFSCD feeding $(1.51 \pm 0.32 \mathrm{U} / \mathrm{l})$ compared with after control diet feeding $(32.71 \pm 6.16 \mathrm{U} / 1 ; P<0.01)$, and the insulin secretion plateau was delayed, indicating that HFSCD feeding induced beta cell dysfunction in minipigs (Fig. 2b).

HOMA-IR indices were significantly higher in the HFSCD group than in the CD group after 2 months feeding, whereas HOMA-beta indices for HFSCD group were only decreased at the end of month 5 (Table 2).

\section{Effect of HFSCD on pancreatic islet}

The majority of pancreatic islets in the HFSCD group were normal as in the control group (Fig. 3B, ×400). A few pancreatic islets in the HFSCD group became atrophied with a loss in number of the islets and cells within them (Fig. 3A, black arrow, ×400). No inflammatory cell infiltration was observed in the pancreas of either of the two groups.

\section{Effect of HFSCD on renal function}

As shown in Table 3, the urinary glucose/creatinine ratio and albumin/creatinine ratio were significantly increased at the 4th month of HFSCD-feeding $(P<0.05)$. Urinary NAG: creatinine ratio and NAG clearance in the HFSCD group were higher (about 1.73- and 1.69fold, respectively) than those in the CD group only at month 5 of the study. No significant changes were observed in creatinine and BUN levels of plasma and

Table 2. Effects of HFSCD on glucose tolerance and beta cell function in Chinese Bama minipigs ( $\mathrm{n}=5)$

\begin{tabular}{|c|c|c|c|c|c|c|}
\hline Indices & 0 & 1 month & 2 months & 3 months & 4 months & 5 months \\
\hline \multicolumn{7}{|c|}{ HOMA-IR } \\
\hline $\mathrm{CD}$ & $1.38 \pm 0.18$ & $1.54 \pm 0.50$ & $1.63 \pm 0.47$ & $1.97 \pm 0.63$ & $1.51 \pm 0.32$ & $1.64 \pm 0.45$ \\
\hline HFSCD & $1.59 \pm 0.26$ & $2.29 \pm 0.39$ & $4.38 \pm 1.05^{*}$ & $7.04 \pm 1.05 \#$ & $10.40 \pm 1.75 \#$ & $7.83 \pm 1.63 \#$ \\
\hline \multicolumn{7}{|c|}{ HOMA-beta } \\
\hline $\mathrm{CD}$ & $168.54 \pm 30.67$ & $128.14 \pm 25.69$ & $176.19 \pm 14.78$ & $170.89 \pm 17.16$ & $171.20 \pm 10.12$ & $136.52 \pm 21.55$ \\
\hline HFSCD & $130.61 \pm 36.44$ & $85.94 \pm 21.94$ & $102.50 \pm 24.72$ & $111.30 \pm 26.31$ & $87.63 \pm 17.65$ & $56.48 \pm 11.48 *$ \\
\hline \multicolumn{7}{|c|}{ AIR (U/L) } \\
\hline $\mathrm{CD}$ & $48.61 \pm 5.72$ & $49.57 \pm 6.81$ & $46.52 \pm 7.77$ & $43.73 \pm 8.40$ & $40.26 \pm 6.63$ & $32.71 \pm 6.16$ \\
\hline HFSCD & $49.24 \pm 5.85$ & $48.63 \pm 7.04$ & $35.83 \pm 5.82$ & $24.39 \pm 6.17 *$ & $13.01 \pm 3.17 \#$ & $1.51 \pm 0.32 \#$ \\
\hline \multicolumn{7}{|c|}{$K_{G}(\% / \min )$} \\
\hline $\mathrm{CD}$ & $2.54 \pm 0.75$ & $2.45 \pm 0.66$ & $2.52 \pm 0.61$ & $2.43 \pm 0.58$ & $2.37 \pm 0.56$ & $1.95 \pm 0.21$ \\
\hline HFSCD & $2.69 \pm 0.70$ & $2.41 \pm 0.58$ & $1.38 \pm 0.47$ & $1.04 \pm 0.26^{*}$ & $-3.26 \pm 0.78 \#$ & $-7.38 \pm 1.36 \#$ \\
\hline
\end{tabular}

Values are means $\pm \mathrm{SD} ; * P<0.05$ HFSCD $v s . \mathrm{CD} ; \# P<0.01$ HFSCD $v s . \mathrm{CD}$.

AIR is the acute insulin response. $K_{G}$ is the glucose elimination constant. 

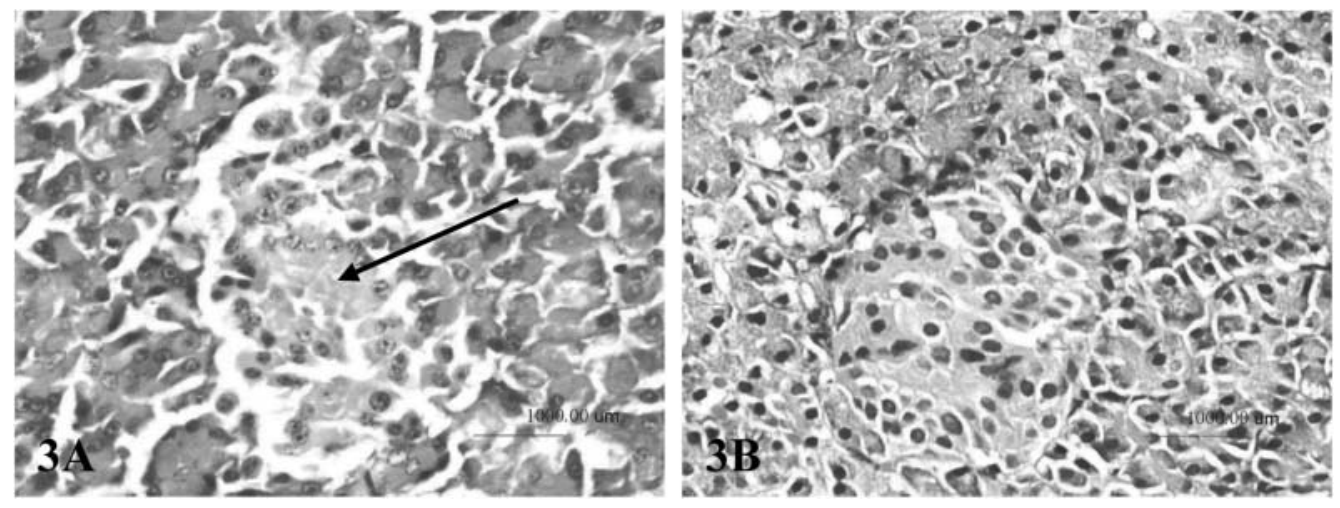

Fig. 3. Effects of the high-fat/high-sucrose/high-cholesterol diet fed to minipigs for 5 months on the morphology of pancreatic islets. A few pancreatic islets from the HFSCD group became atrophied with a loss in the number of the islets and cells within them (Fig. 3A, black arrow, in HE staining, $\times 400$ ) compared with control group (Fig. 3B, $\times 400$ ). No inflammatory cell infiltration was observed in the pancreas of either of the two groups.

urine between HFSCD and CD pigs.

\section{Effect of HFSCD on renal morphological changes}

There was no significant difference in the left kidney weight index between the two groups $(\mathrm{CD}, 3.42 \pm 0.51$ $\mathrm{g} / \mathrm{kg} v s . \mathrm{HFSCD}, 2.89 \pm 0.71 \mathrm{~g} / \mathrm{kg}, P=0.259)$. Histological analysis of the kidneys in HFSCD-fed minipigs with HE-staining revealed that the predominant form of glomerular alteration is hypertrophy with hypercellularity (black arrow) and focal segmental glomerulosclerosis with destroyed structure of the glomerular capsule (white arrow) (Fig. 4A, ×400). Some distal tubules and macula densae were vacuolated and degenerated, accompanied with inflammatory cell infiltration. Masson trichrome staining of the kidneys of the HFSCD group showed an overt increase in the mesangial area and proliferation of collagen fiber (Fig. $4 \mathrm{C}$, blue dye, $\times 400$ ). There were no obvious changes in renal histology of CD-fed minipigs (Fig. 4B, 4D, $\times 400$ ). The grade of the glomerulosclerosis index was statistically significantly higher in the HFSCD group than that in the CD group $(1.40 \pm 0.21$ vs. $0.45 \pm 0.14$, $P=0.001$ ), representing on average, moderate damage to all glomeruli (Table 4).

Type IV collagen, a major component of basement membranes and extracellular matrix, was overexpressed in the glomeruli and tubulointerstitium of the HFSCD pigs (Fig. 4E, $\times 400$, brown staining, same as below) compared with the CD pigs (Fig. 4F, $\times 400$ ). Tubular and glomerular expression of CTGF was increased in the HFSCD pigs (Fig. 4G, $\times 600$ ) in comparison with the $\mathrm{CD}$ pigs (Fig. $4 \mathrm{H}, \times 400$ ). Immunoreactivity of MMP-2 showed a significant decrease in the HFSCD group (Fig. 4I, $\times 400$ ) vis-à-vis the CD group (Fig. 4J, $\times 400)$.

\section{Discussion}

This study characterizes the high-fat/high-sucrose/ high-cholesterol diet-fed minipig as a robust model for impaired glucose tolerance (IGT) and early-stage type 2 diabetes-associated nephropathy. In the present study the data for Chinese Bama minipigs show that HFSCD results in: (1) increased body weight gain; (2) stable hyperglycemia but progressive hyperinsulinemia with significantly increased HOMA-IR index but reduced glucose elimination, indicating progressive worsening of insulin resistance; (3) damaged islet structure and impaired insulin secretion by the results of HOMAbeta index and OGTT (particularly AIR), suggesting beta-cell dysfunction; (4) early-onset alteration of renal function including microalbuminuria, glucosuria and elevated excretion of urinary NAG, and excessive deposition of extracellular matrix inducing moderate glomerulosclerosis.

The high-fat/high-sucrose/high-cholesterol diet caused a number of perturbances of homeostasis in miniature swine including a progressive increase in fasting plasma glucose and insulin concentrations accompanied by augmented plasma total cholesterol and triglycer- 
Table 3. Effects of HFSCD on renal function in Chinese Bama minipigs ( $n=5)$

\begin{tabular}{|c|c|c|c|c|}
\hline Parameters & 0 & 2 months & 4 months & 5 months \\
\hline \multicolumn{5}{|c|}{ Urinary glucose/creatinine ratio $(\mathrm{mmol} / \mathrm{mmol})$} \\
\hline $\mathrm{CD}$ & $0.0365 \pm 0.0057$ & $0.0397 \pm 0.0098$ & $0.0411 \pm 0.0054$ & $0.0408 \pm 0.0078$ \\
\hline HFSCD & $0.0378 \pm 0.0066$ & $0.0429 \pm 0.0085$ & $0.0625 \pm 0.0079 *$ & $0.0662 \pm 0.0107 \#$ \\
\hline \multicolumn{5}{|c|}{ Urinary albumin/creatinine ratio $(\mathrm{mg} / \mathrm{g})$} \\
\hline $\mathrm{CD}$ & $12.26 \pm 2.54$ & $12.77 \pm 3.33$ & $13.52 \pm 4.67$ & $12.60 \pm 3.27$ \\
\hline HFSCD & $12.73 \pm 3.14$ & $14.16 \pm 3.53$ & $25.13 \pm 4.65^{*}$ & $48.08 \pm 11.77 \#$ \\
\hline \multicolumn{5}{|c|}{ Urinary NAG/creatinine ratio $(\mathrm{U} / \mathrm{g})$} \\
\hline $\mathrm{CD}$ & $7.15 \pm 2.27$ & $6.37 \pm 1.91$ & $6.10 \pm 1.87$ & $6.28 \pm 1.58$ \\
\hline HFSCD & $6.44 \pm 1.87$ & $6.47 \pm 1.35$ & $7.05 \pm 1.64$ & $10.87 \pm 1.71 \#$ \\
\hline \multicolumn{5}{|c|}{ NAG clearance } \\
\hline $\mathrm{CD}$ & $6.27 \pm 1.55$ & $7.12 \pm 2.27$ & $7.73 \pm 1.62$ & $8.69 \pm 1.29$ \\
\hline HFSCD & $7.93 \pm 1.37$ & $6.51 \pm 1.72$ & $9.86 \pm 2.11$ & $14.72 \pm 3.39 *$ \\
\hline \multicolumn{5}{|c|}{ Plasma creatinine $(\mu \mathrm{mol} / \mathrm{l})$} \\
\hline $\mathrm{CD}$ & $28.56 \pm 5.54$ & $28.97 \pm 7.44$ & $28.81 \pm 6.02$ & $29.69 \pm 3.81$ \\
\hline HFSCD & $26.67 \pm 6.53$ & $27.71 \pm 6.36$ & $30.76 \pm 8.91$ & $33.38 \pm 9.10$ \\
\hline \multicolumn{5}{|c|}{ Urinary creatinine $(\mathrm{mmol} / \mathrm{l})$} \\
\hline $\mathrm{CD}$ & $3.77 \pm 0.53$ & $4.08 \pm 0.84$ & $4.13 \pm 1.00$ & $4.43 \pm 0.87$ \\
\hline HFSCD & $4.05 \pm 0.72$ & $4.20 \pm 0.92$ & $4.08 \pm 0.76$ & $4.71 \pm 0.69$ \\
\hline \multicolumn{5}{|c|}{ Plasma BUN (mmol/l) } \\
\hline $\mathrm{CD}$ & $5.89 \pm 1.05$ & $6.20 \pm 1.77$ & $6.31 \pm 1.36$ & $6.57 \pm 1.24$ \\
\hline HFSCD & $6.06 \pm 1.10$ & $6.35 \pm 1.07$ & $6.55 \pm 1.87$ & $6.79 \pm 1.58$ \\
\hline \multicolumn{5}{|c|}{ Urinary BUN (mmol/l) } \\
\hline $\mathrm{CD}$ & $206.67 \pm 32.36$ & $220.46 \pm 39.23$ & $217.45 \pm 32.31$ & $231.03 \pm 40.47$ \\
\hline HFSCD & $221.74 \pm 40.38$ & $215.35 \pm 41.62$ & $238.62 \pm 61.24$ & $223.49 \pm 50.13$ \\
\hline
\end{tabular}

Values are means $\pm \mathrm{SD} ; * P<0.05$ HFSCD $v s . \mathrm{CD} ; \# P<0.01$ HFSCD $v s . \mathrm{CD}$.

NAG is N-acetyl-beta-glucosaminidase. BUN is blood urea nitrogen.

ides. The results of AIR and $K_{G}$ from OGTT revealed an obvious suppression of insulin secretion and marked deterioration of glucose elimination at 3 months after the start of HFSCD. Moreover, HOMA-IR indices significantly increased in the HFSCD group from the second month, which longitudinally reflects aggravated insulin resistance, whereas HOMA-beta indices were obviously decreased only at month 5 , when the level of fasting insulin tended to drop off. Thus, it is tempting to speculate from these observations that after feeding HFSCD, exogenous hyperglycaemia and dyslipidaemia cause a progressive decline in insulin sensitivity at an early stage. Subsequently, insulin secretion increases gradually to compensate for insulin resistance and hyperglycaemia, and then beta-cell failure results in the reduction of baseline insulin due to maladaptation of the beta cells caused by glucotoxicity and lipotoxicity. Hence, the model shows two important mechanistic characteristics of IGT and type 2 diabetes: insulin resistance and islet dysfunction, which is very similar to the human pathophysiology of type 2 diabetes [3].

In this study, we also show that the model is suitable for study of diabetic nephropathy because humans and pigs share important similarities in the renal anatomy, physiology and pathophysiology [14]. The excretion of urinary albumin in HFSCD pigs, which is a parameter of glomerular and tubular alterations, increased after 4 months of feeding with HFSCD. Measurement of the protein:creatinine ratio in a single morning urine specimen is an excellent alternative to measurement of protein in 24-h urine collection [11]. As a marker of tubular injury, we assessed the total urinary activity of NAG, a lysosomal enzyme of tubular origin [1]. NAG activity was significantly higher in animals of the HFSCD group, indicating an initial malfunction of the proximal tubular epithelial cells. Creatinine and BUN 


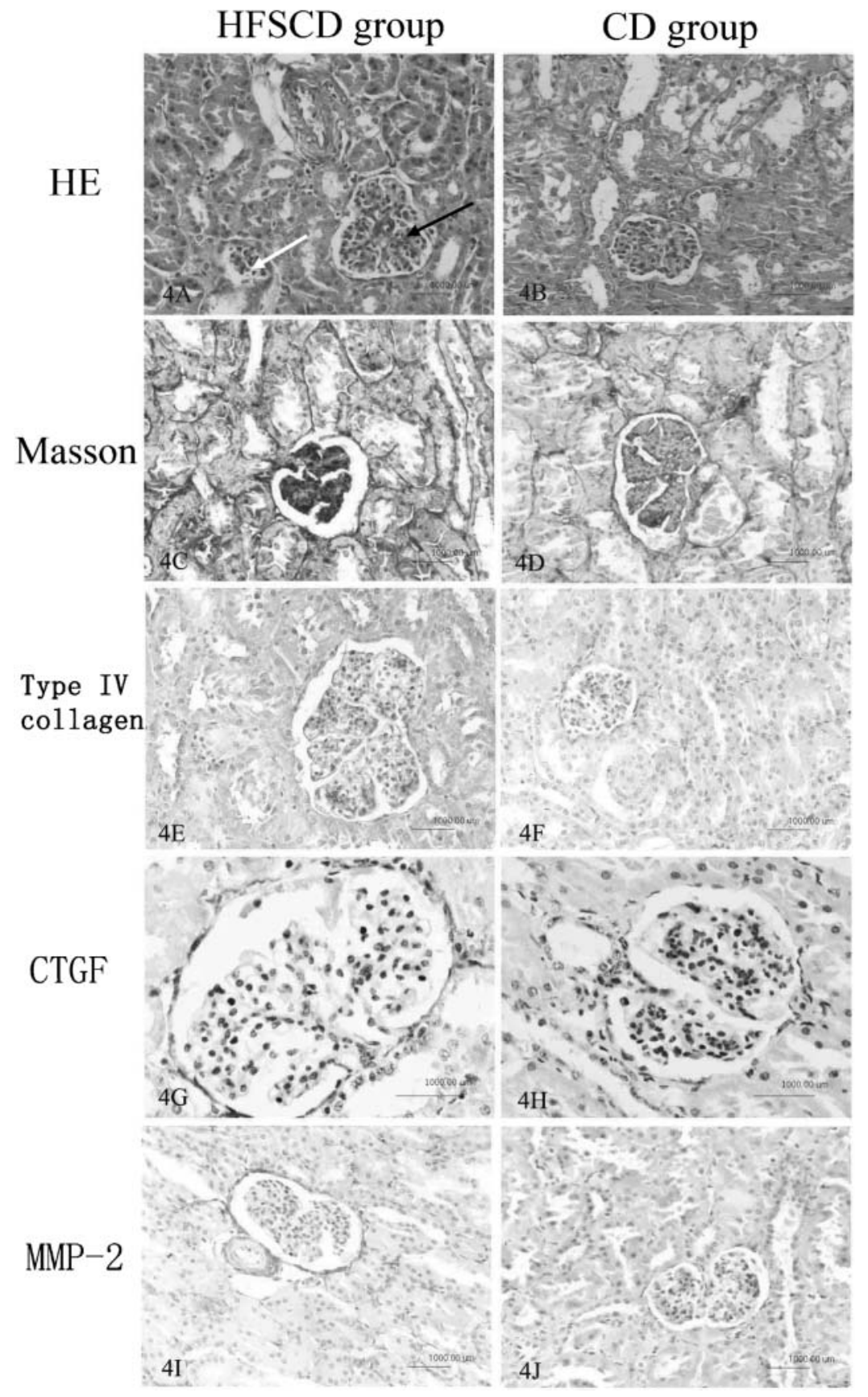

Fig. 4. Effects of the high-fat/high-sucrose/high-cholesterol diet fed to minipigs for 5 months on the renal morphology. Compared with the CD group (Fig. 4B, 4D), glomeruli of the HFSCD group showed hypertrophy with hypercellularity (black arrow), atrophy with destroyed structure of the glomerular capsule (white arrow) (Fig. 4A, in HE staining, $\times 400$ ), an expanded mesangial area and proliferation of collagen fiber (Fig. 4C, blue dye, Masson trichrome staining, $\times 400$ ). The protein expression of type IV collagen (brown staining, same as below) was increased in the glomerulus and tubulointerstitium in HFSCD-fed minipigs (Fig. 4E, $\times 400$ ) compared with that in control minipigs (Fig. 4F, immunostaining for type IV collagen, $\times 400$ ). More intensive expression of connective tissue growth factor (CTGF) in epithelial cells of Bowman's capsule and distal convoluted tubules were observed in the HFSCD group (Fig. 4G, ×600) than in the CD group (Fig. $4 \mathrm{H}$, immunostaining for CTGF, $\times 600)$, whereas the intensity of matrix metalloproteinase-2 (MMP-2) immunostaining was decreased in the glomerulus and tubulointerstitium of HFSCD-fed minipigs (Fig. 4I, $\times 400$ ) compared with that in control minipigs (Fig. 4J, immunostaining for MMP-2, ×400). 
in plasma and urine of HFSCD-fed pigs, which are important parameters in the assessment of end-stage renal failure, did not worsen within the experimental period as compared with the control group.

The present study characterized the morphological features of a diet-induced miniature swine model that exhibits hypertrophy of the glomeruli, proliferation of collagen fiber, expansion of the mesangial matrix, and moderate glomerulosclerosis. In addition, overexpression of type IV collagen occurred in glomeruli and tubulointerstitium in minipigs of the HFSCD group, implying that extracellular matrix proteins excessively accumulated in the mesangium and basement membrane of the glomeruli and in the renal tubulointerstitium. Regulation of extracellular matrix formation is a dynamic phenomenon involving synthetic and degradative processes. The former involves the induction of various growth factors such as CTGF [9]. CTGF is a crucial mediator for transforming growth factor-beta-stimulated extracellular matrix synthesis (e.g. type IV collagen, fibronectin and laminin) [9]. The latter involves matrix metalloproteinases (MMPs), the major factors that degrade extracellular matrix and display substrate specificity. MMP-1 and -13 degrade interstitial collagens, whereas MMP-2 and -9 degrade type IV collagen and fibronectin [10]. In our study, increased expression of type IV collagen and CTGF and decreased immunoreactivity of MMP-2 in HFSCD-fed pigs indicated that HFSCD might not only promote extracellular matrix deposition and renal fibrosis through increasing the production of fibrogenic factors such as CTGF but might also attenuate extracellular protein degradation and removal by inhibiting the expression of MMP-2. These pathological features in the kidneys of pigs are similar to those of human diabetic nephropathy [4].

In conclusion, we have shown here that the Chinese Bama miniature swine fed with high-fat/high-sucrose/ high-cholesterol diet for 5 months is prone to insulin resistance and type 2 diabetes, and is a robust and valuable model for early-stage diabetic nephropathy. It may therefore be used both for studying the pathogenic features of diabetogenic diet and for investigating the pathogenesis of diabetic nephropathy. We also showed specifically that the diet increased the protein expression of CTGF, promoted excessive extracellular matrix accumulation, and suppressed extracellular protein degradation, eventually resulting in moderate
Table 4. Effect of HFSCD on glomerulosclerosis index in Chinese Bama minipigs $(\mathrm{n}=5)$

\begin{tabular}{lccccc}
\hline Group & Grade 0 & Grade 1 & Grade 2 & Grade 3 & Grade 4 \\
\hline CD & 3 & 2 & 0 & 0 & 0 \\
HFSCD & 0 & 2 & 3 & 0 & 0 \\
\hline
\end{tabular}

glomerulosclerosis in the minipig kidney.

\section{Acknowledgments}

This study was supported by Project 973 of China (G2000056905) and the National Natural Sciences Foundation of China (30370675) and Hunan Provincial Natural Science Foundation of China (04JJ3058 and 05JJ20006). The authors gratefully acknowledge the pathological assistance of Prof. Xiutian Zhou, Mr. Rongjun Tang and Mrs. Ying Song (Department of Pathology, Nanhua University, Hengyang, Hunan, P. R. China).

\section{References}

1. Bazzi, C., Petrini, C., Rizza, V., Arrigo, G., Napodano, P., Paparella, M., and D'Amico, G. 2002. Urinary N-acetylbeta-glucosaminidase excretion is a marker of tubular cell dysfunction and a predictor of outcome in primary glomerulonephritis. Nephrol. Dial. Transplant. 17: 18901896.

2. Bonora, E., Targher, G., Alberiche, M., Bonadonna, R.C., Saggiani, F., Zenere, M.B., Monauni, T., and Muggeo, M. 2000. Homeostasis model assessment closely mirrors the glucose clamp technique in the assessment of insulin sensitivity: studies in subjects with various degrees of glucose tolerance and insulin sensitivity. Diabetes Care 23: 57-63.

3. Fujimoto, W.Y. 2000. The importance of insulin resistance in the pathogenesis of type 2 diabetes mellitus. Am. J. Med. 108: 9S-14S.

4. Gross, J.L., de Azevedo, M.J., Silveiro, S.P., Canani, L.H., Caramori, M.L., and Zelmanovitz, T. 2005. Diabetic nephropathy: diagnosis, prevention, and treatment. Diabetes Care 28: 164-176.

5. Huang, B.W., Chiang, M.T., Yao, H.T., and Chang, W. 2004. The effect of high-fat and high-fructose diets on glucose tolerance and plasma lipid and leptin levels in rats. Diabetes Obes. Metab. 6: 120-126.

6. Kasiske, B.L., O’Donnell, M.P., Schmitz, P.G., Kim, Y., and Keane, W.F. 1990. Renal injury of diet-induced hypercholesterolemia in rats. Kidney Int. 37: 880-891.

7. Kluwe, W.M. 1981. Rapid, automated measurements of 
urinary protein and glucose concentrations. J. Pharmacol. Methods 5: 235-240.

8. Larsen, M.O., Rolin, B., Wilken, M., Carr, R.D., and Svendsen, O. 2002. High-fat high-energy feeding impairs fasting glucose and increases fasting insulin levels in the Göttingen minipig: results from a pilot study. Ann. N.Y. Acad. Sci. 967: 414-423.

9. Mason, R.M. and Wahab, N.A. 2003. Extracellular matrix metabolism in diabetic nephropathy. J. Am. Soc. Nephrol. 14: 1358-1373.

10. Matrisian, L.M. 1990. Metalloproteinases and their inhibitors in matrix remodeling. Trends Genet. 6: 121-125.

11. Nathan, D.M., Rosenbaum, C., and Protasowicki, V.D. 1987. Single-void urine samples can be used to estimate quantitative microalbuminuria. Diabetes Care 10: 414-418.

12. Popov, D., Simionescu, M., and Shepherd, P.R. 2003. Saturated-fat diet induces moderate diabetes and severe glomerulosclerosis in hamsters. Diabetologia 46: 1408-1418.

13. Saito, T., Sumithran, E., Glasgow, E.F., and Atkins, R.C. 1987. The enhancement of aminonucleoside nephrosis by the co-administration of protamine. Kidney Int. 32: 691-699.

14. Samstein, B. and Platt, J. 2001. Physiologic and immunologic hurdles to xenotransplantation. J. Am. Soc. Nephrol. 12: 182-193.

15. Wallace, T.M., Levy, J.C., and Matthews, D.R. 2004. Use and abuse of HOMA modeling. Diabetes Care 27: 1487-
1495.

16. Wang, P.R., Guo, Q., Ippolito, M., Wu, M., Milot, D., Ventre, J., Doebber, T., Wright, S.D., and Chao, Y.S. 2001. High fat fed hamster, a unique animal model for treatment of diabetic dyslipidemia with peroxisome proliferator activated receptor alpha selective agonists. Eur. J. Pharmacol. 427: 285-293.

17. Wei, P., Lane, P.H., Lane, J.T., Padanilam, B.J., and Sansom, S.C. 2004. Glomerular structural and functional changes in a high-fat diet mouse model of early-stage Type 2 diabetes. Diabetologia 47: 1541-1549.

18. Wilson, S.H., Chade, A.R., Feldstein, A., Sawamura, T., Napoli, C., Lerman, A., and Lerman, L.O. 2003. Lipidlowering-independent effects of simvastatin on the kidney in experimental hypercholesterolaemia. Nephrol. Dial. Transplant. 18: 703-709.

19. Winzell, M.S. and Ahren, B. 2004. The high-fat diet-fed mouse: a model for studying mechanisms and treatment of impaired glucose tolerance and type 2 diabetes. Diabetes 53: S215-S219.

20. Xi, S., Yin, W., Wang, Z., Kusunoki, M., Lian, X., Koike, T., Fan, J., and Zhang, Q. 2004. A minipig model of highfat/high-sucrose diet-induced diabetes and atherosclerosis. Int. J. Exp. Path. 85: 223-231. 